\title{
Avaliação do Perfil Radial do Lenho de Árvores de Teca (Tectona grandis L.f.) Através da Tomografia de Impulso
}

\author{
Vinicius Resende de Castro ${ }^{1}$, Mário Tomazello Filho ${ }^{1}$, Marco Aurelio Arizapana ${ }^{1}$, \\ José de Castro Silva ${ }^{2}$, Demóstenes Ferreira da Silva Filho ${ }^{1}$, \\ Jefferson Lordello Polizel ${ }^{1}$, Ugo Leandro Belini ${ }^{1}$
}

${ }^{1}$ Departamento de Ciências Florestais, Escola Superior de Agricultura "Luiz de Queiroz" - ESALQ, Universidade de São Paulo - USP

${ }^{2}$ Departamento de Engenharia Florestal, Universidade Federal de Viçosa - UFV

\section{RESUMO}

A tomografia de impulso constitui-se em uma das mais importantes metodologias de análise não destrutiva, avaliando a velocidade das ondas mecânicas através do lenho e gerando imagens da seção transversal do tronco das árvores. No presente trabalho, foi aplicado o tomógrafo de impulso no lenho de 20 árvores de Tectona grandis e foram analisadas as imagens tomográficas. Os resultados evidenciaram: i) a geração de ondas mecânicas com velocidade média no lenho de $1.087 \mathrm{~m} / \mathrm{s}$; ii) um coeficiente de variação de 19,81\%, de baixa variabilidade, demonstrando a eficácia do método; e iii) os defeitos internos no lenho, detectados nas imagens tomográficas através da diferença de cor.

Palavras-chave: velocidade de onda, qualidade da madeira, método não destrutivo.

\section{Evaluation of the Radial Profile of Teak Tree Wood (Tectona grandis L.f.) Through Impulse Tomography}

\begin{abstract}
Tomography constitutes one of the most important non-destructive methods of non-destructive analysis, evaluating the velocity of mechanical waves through the wood and producing images of the radial profile of teak wood by means of impulse. In this paper, the impulse tomography was applied in the wood of 20 Tectona grandis trees and the images were analyzed. The results showed: i) the generation of mechanical waves in wood with mean speed of $1,087 \mathrm{~m} / \mathrm{s}$; ii) the variation coefficient of $19.81 \%$, with low variability, demonstrating the effectiveness of the method; and iii) the internal defects in wood detected in tomography images through color difference.
\end{abstract}

Keywords: velocity wave, quality wood, non-destructive method. 


\section{INTRODUÇÃO}

As técnicas não destrutivas de análise do tronco das árvores em pé nas florestas urbanas possibilitam detectar os defeitos internos do lenho, prevendo o risco de queda e propiciando o manejo correto pela poda de galhos afetados por organismos xilófagos, aumentando sua longevidade e a sanidade biológica (Ferreira, 2009). Nas plantações florestais, tais técnicas permitem a avaliação da qualidade do lenho e a sua caracterização para aplicação como madeira sólida e seus produtos, bem como no manejo florestal sustentado e na seleção nos programas de melhoramento florestal (Pereira, 2009). Atualmente, dentre os métodos não destrutivos, destaca-se o tomógrafo de impulso (Pereira et al., 2007), que permite a reconstrução de seções transversais do tronco e dos galhos das árvores, tornando possível delimitar e quantificar o lenho biodegradado e inferir sobre algumas de suas propriedades. A imagem da estrutura interna do lenho gerada pelo tomógrafo de impulso, em resposta à diferença da velocidade de transmissão das ondas sonoras, pode ser correlacionada com as propriedades físico-químicas (densidade, módulo de elasticidade, umidade, etc) e possibilita determinar a percentagem de cerne/ alburno, madeira de reação, nós, podridão do lenho, excentricidade da medula, etc. (Rinntech, 2005; Picus, 2009).

O tomógrafo de impulso baseia-se na medição do tempo de transmissão das ondas entre os sensores e sua discriminação em faixas através de software. A velocidade da onda depende do estado de sanidade do lenho, sendo que fraturas, cavidades e podridão reduzem o tempo de transmissão das ondas, formando uma imagem bidimensional da seção transversal do tronco codificada em tonalidades de diferentes cores (Rinntech, 2005; Picus, 2009).

O tomógrafo de impulso foi utilizado na avaliação do tronco de árvores de Quercus alba e de Carya spp., mostrando correlação com a avaliação visual, com erro de 3 a $8 \%$ na imagem produzida (Gilbert \& Smiley, 2004). Da mesma forma, constitui-se em ferramenta eficiente para estimar a densidade do lenho de árvores de Caesalpinia echinata, Schizolobium parayba e Eucaliptus saligna, com similaridade com os dados de densidade por densitometria de raios X (Pereira, 2009). A comparação dos resultados da análise do lenho das árvores por tomografia de impulso indicou que dados de melhor qualidade da sanidade foram gerados, em relação ao resistógrafo (Rollo, 2009). $\mathrm{Na}$ análise do lenho de árvores de Tectona grandis, a tomografia de impulso permitiu diagnosticar regiões com degradação do lenho (Amodei et al., 2010).

Pelo exposto, o presente trabalho teve por objetivo avaliar o perfil radial do lenho do tronco de árvores adultas de Tectona grandis, utilizando a metodologia de tomografia de impulso.

\section{MATERIAL E MÉTODOS}

\subsection{Seleção das árvores e obtenção das imagens tomográficas}

Foram selecionadas 20 árvores de teca, com 41 anos, de uma plantação localizada no Campus da ESALQ-USP, nas coordenadas de $22^{\circ} 42^{\prime} \mathrm{S}$ de latitude, $47^{\circ} 38^{\prime} \mathrm{O}$ de longitude e $540 \mathrm{~m}$ de altitude. $\mathrm{Na}$ altura do DAP do tronco das árvores, foram fixados 11 pregos metálicos equidistantes, a $2 \mathrm{~cm}$ de profundidade, atingindo a região do alburno. Nos pregos metálicos, foram inseridos os sensores do tomógrafo de impulso marca Arbotom, modelo 1.73a e, em seguida, foi instalado um cabo, conectando todos os pregos com o primeiro sensor ligado à bateria (Figura 1). Em cada sensor, foram

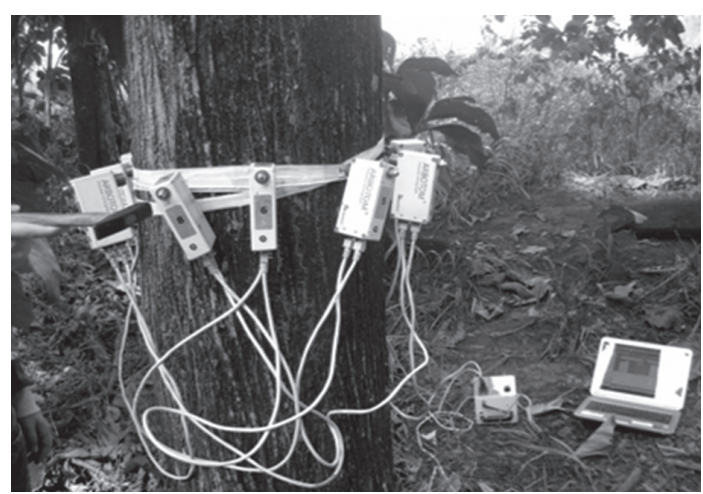

Figura 1. Tomógrafo de impulso instalado no tronco de árvore de teca observando-se o momento das batidas para aquisição dos dados.

Figure 1. Impulse tomography installed in the trunk of teak tree observing the moment of beats for data acquisition. 
executadas cinco batidas com um martelo, podendo aumentar em número, até que a percentagem de erro atingisse a faixa de valor aceitável (entre 0 e $10 \%)$, de acordo com a Tabela Delta \%, do software Arbotom 1.73a. A velocidade das ondas que atinge os diferentes sensores é calculada por um software, gerando um gráfico de velocidade de onda da seção transversal e produzindo uma imagem tomográfica da seção transversal do tronco das árvores de teca.

\subsection{Confecção dos gráficos do perfil radial do tronco das árvores}

Para a análise do perfil radial, foram utilizadas imagens no modelo de cor rainbow, com resolução de superfície 2D igual a $1 \mathrm{~mm}$ (opção gráfica fornecida pelo software do equipamento). A partir da imagem, foi selecionada uma faixa da imagem tomográfica, contendo a maior variedade de cores entre os sensores (foram selecionadas, em geral, as dos sensores 5 a 11). A faixa foi analisada por uma linha de pixels com o software Adobe Photoshop, reconhecendo-se os valores RGB de cada pixel e, em seguida, associando-os ao valor de velocidade de onda a cada valor de RGB. O comportamento da velocidade em relação às cores da imagem composta foi analisado segundo Rollo (2009). Os dados foram analisados através da estatística descritiva, observando a variação de velocidade de onda entre as árvores pelo pacote estatístico SPSS.

\section{RESULTADOS E DISCUSSÃO}

As imagens tomográficas geradas pelo software Arbotom $1.73 \mathrm{c}$, referentes à estrutura interna do lenho de pinus e teca, indicaram sintomas de lesões internas (Figura 2). Tal conclusão foi obtida em decorrência da queda na velocidade de propagação das ondas mecânicas entre os sensores. Em algumas árvores, verificou-se que tais conclusões não correspondiam à realidade, uma vez que a grande proximidade entre os sensores mascaravam os resultados, principalmente nas regiões mais periféricas. Em tais situações, a análise se concentrou na área central da amostra.

Verificou-se uma grande variabilidade de cores, como resposta à heterogeneidade do material analisado, envolvendo vários fatores, como idade
(41 anos), ausência de práticas de manejo florestal, usos anteriores para fins acadêmicos e de pesquisa. Nas porções mais centrais das imagens, constatouse uma coloração esverdeada e, em algumas regiões da periferia, uma coloração azulada. Concluiu-se que havia uma menor velocidade de onda no centro, tendendo a um aumento em direção à casca. Algumas árvores apresentaram manchas na porção mais central, que variaram do vermelho ao violeta, evidenciando as menores velocidades de onda, possivelmente devido à natureza do material ali existente e a problemas de sanidade.

Das árvores analisadas, apenas a de $\mathrm{n}^{\circ} .32$ apresentou lenho com tecido considerado anormal, com lesão evidenciada pela coloração rosada, confirmada pela análise visual do seu tronco, no qual se evidenciava a formação de um cancro.

As imagens geradas pelo tomógrafo de impulso na análise do lenho das árvores (Figura 2) indicam o modelo de cor rainbow e resolução de superfície $2 \mathrm{D}$ igual a $1 \mathrm{~mm}$, com as linhas pretas em diagonal evidenciando a faixa de pixels selecionada para a construção do perfil radial. As tonalidades em vermelho, laranja e amarelo indicam menor velocidade de propagação da onda mecânica no lenho das árvores; por outro lado, azul e verde representam a região do lenho com maior velocidade de propagação da onda mecânica.

A construção do perfil radial permite a localização dos defeitos do lenho das árvores, bem como a posição da medula, constituída por tecido parenquimático com maior resistência à passagem das ondas. A variação radial indica, também, diferenças anatômico-físicas nas regiões interna e externa do lenho das árvores de teca, importante na avaliação da sua qualidade e aplicação como madeira sólida (Figura 3).

A análise do perfil radial da densidade aparente do lenho das 20 árvores de teca (Figura 3) proporciona inferir sobre o aumento da velocidade de onda no sentido medula-casca e a delimitação de duas regiões do tronco: a central (com menor velocidade, com madeira juvenil) e a periférica (com maior velocidade, com madeira adulta).

Os valores de velocidade de onda no lenho das árvores de teca (Tabela 1) indicam diferenças, com os maiores valores de velocidade máxima, mínima e 


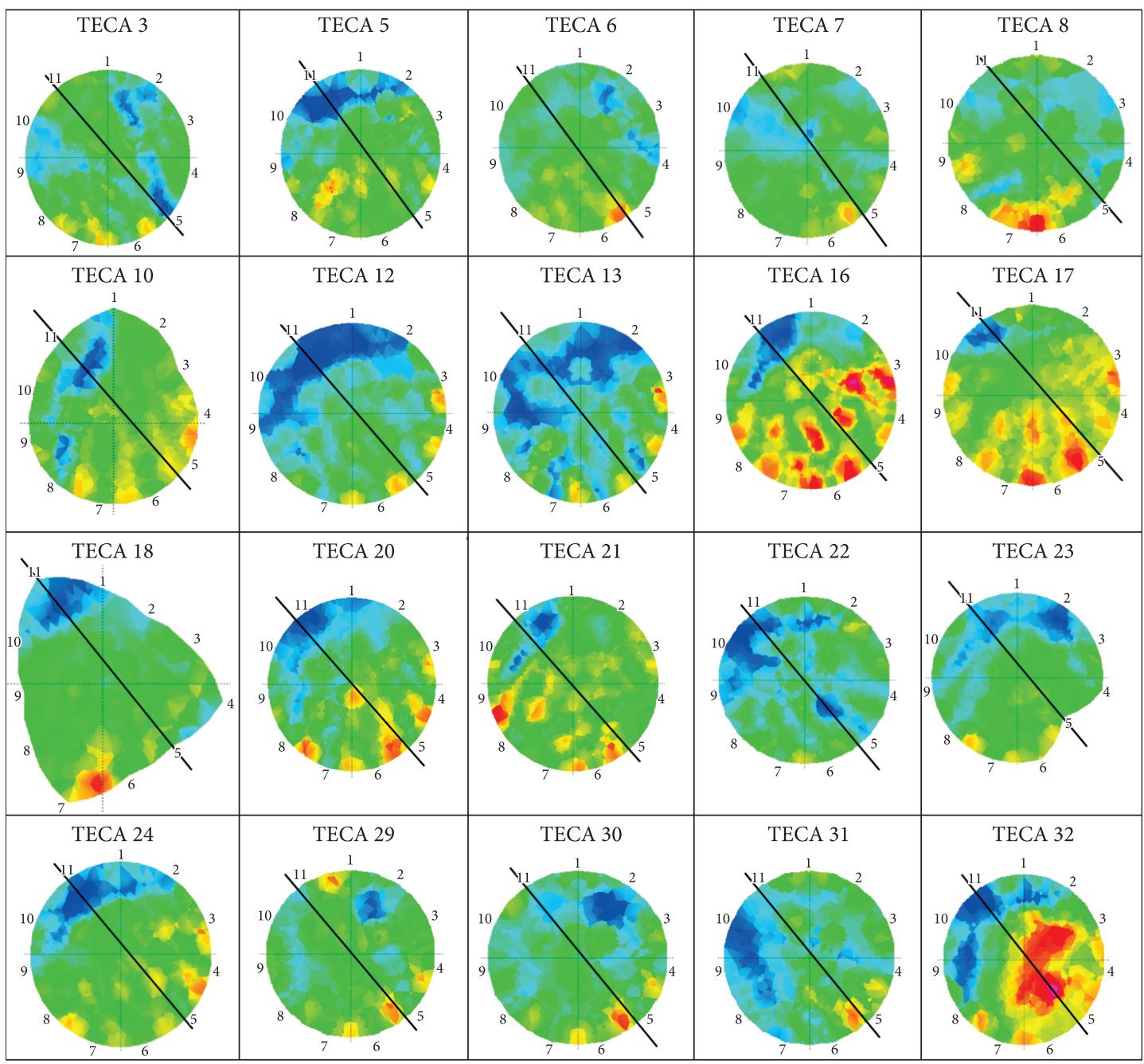

Figura 2. Imagens tomográficas de seções transversais do tronco das árvores de teca.

Figure 2. Tomographic images of the trunk cross sections of teak trees.

média de 1899, 1200 e 1509,09 m/s, respectivamente, obtidos na árvore $\mathrm{n}^{\circ}$. 18. Por outro lado, a árvore de teca $\mathrm{n}^{\circ} .32$ mostrou lenho com velocidade média de $772,12 \mathrm{~m} / \mathrm{s}$, desvio padrão $247 \mathrm{~m} / \mathrm{s}$ e elevado coeficiente de variação de 31,99\%, com amplitude de faixa de $758 \mathrm{~m} / \mathrm{s}$, ampla em relação às faixas das demais árvores. A árvore de teca ${ }^{\circ} .8$ apresentou o menor coeficiente de variação $(3,12 \%)$, com variação da velocidade de 1007 a $1131 \mathrm{~m} / \mathrm{s}$, média $1070,05 \mathrm{~m} / \mathrm{s}$ e desvio padrão de 33,42 . Verifica-se que, de modo geral, o menor valor do coeficiente de variação no perfil analisado corresponde ao lenho da árvore de teca com melhor estado de sanidade. Os resultados obtidos para as árvores de teca indicam que a média da velocidade das ondas no lenho foi de $1087,13 \mathrm{~m} / \mathrm{s}$, com o desvio padrão de $211,04 \mathrm{~m} / \mathrm{s}$ e o coeficiente de variação de $19,41 \%$, evidenciando baixa variabilidade e demonstrando a eficácia e a potencialidade da utilização do equipamento e do método de tomografia de impulso na avaliação do lenho do tronco das árvores.

A velocidade de onda no interior do tronco das árvores de teca apresenta uma distribuição assimétrica positiva (Tabela 2, Figura 4), podendo-se concluir que a moda é maior que a mediana e a média; em cerca de $50 \%$ das árvores, a velocidade de onda através do lenho encontra-se nos intervalos de 907-1007 e de $1007-1107 \mathrm{~m} / \mathrm{s}$. 
TECA 3

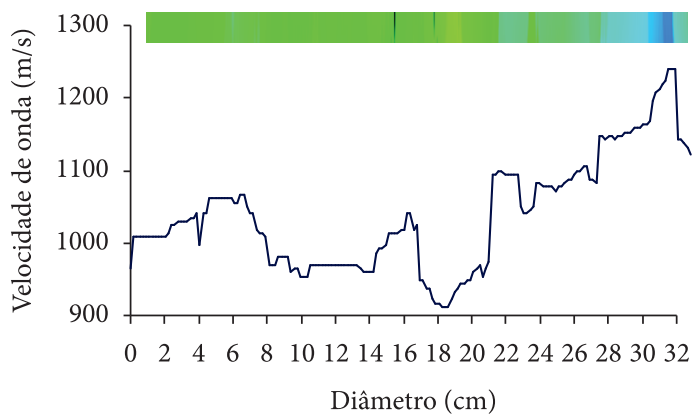

TECA 6

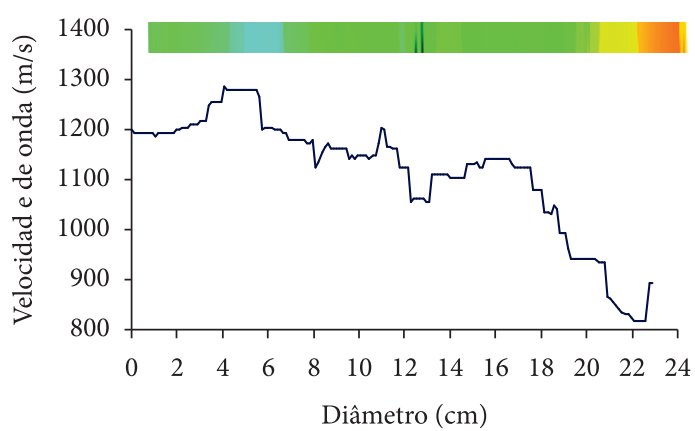

TECA 8

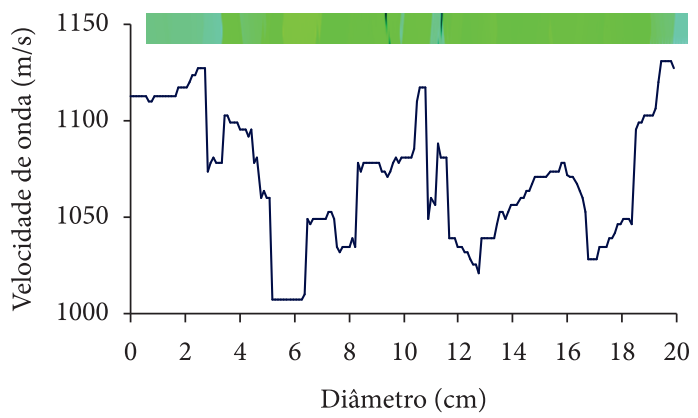

TECA 12

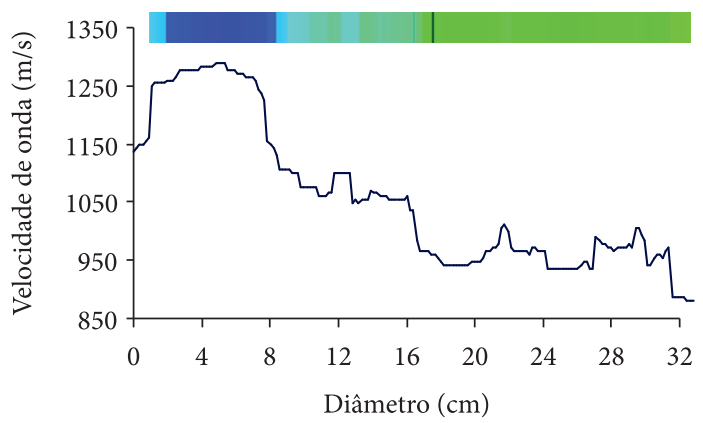

Figura 3. Perfil radial do lenho das 20 árvores de teca. Figure 3. Wood radial profile of the 20 teak trees.

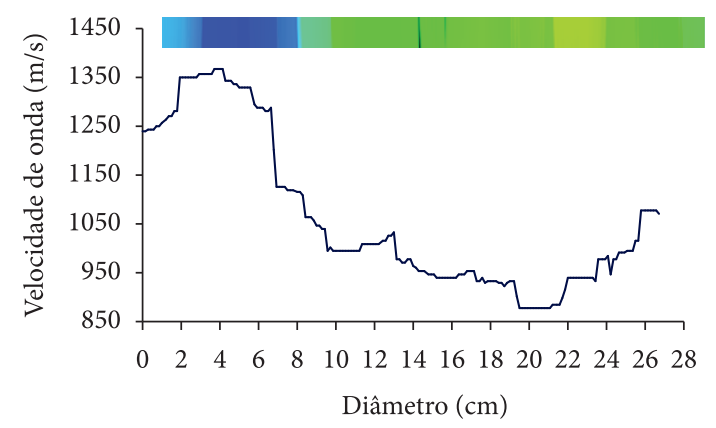

TECA 7
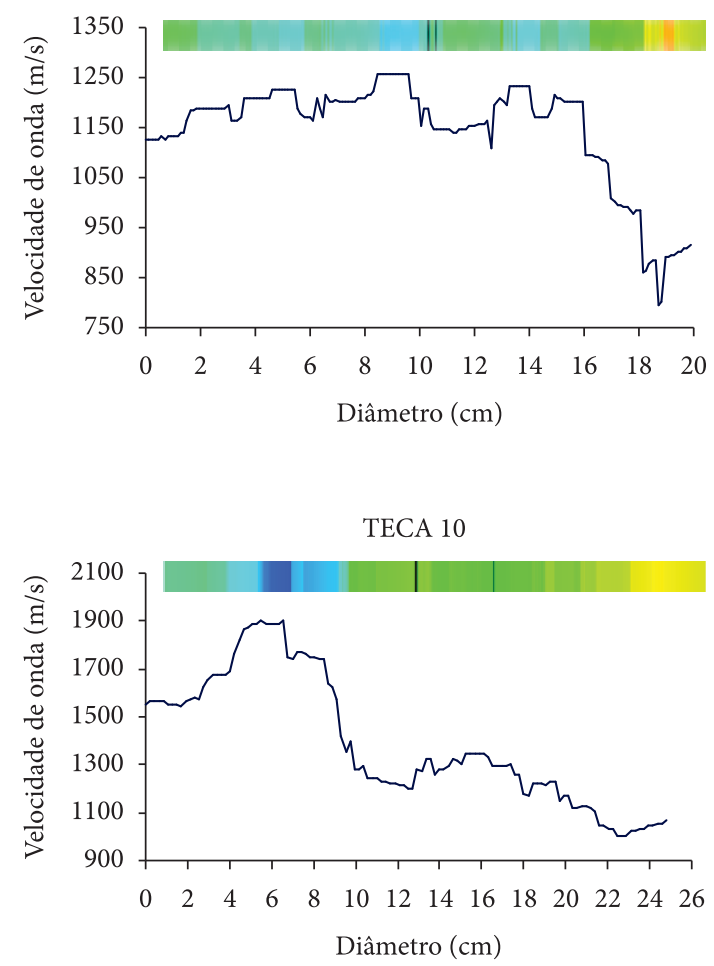

TECA 13

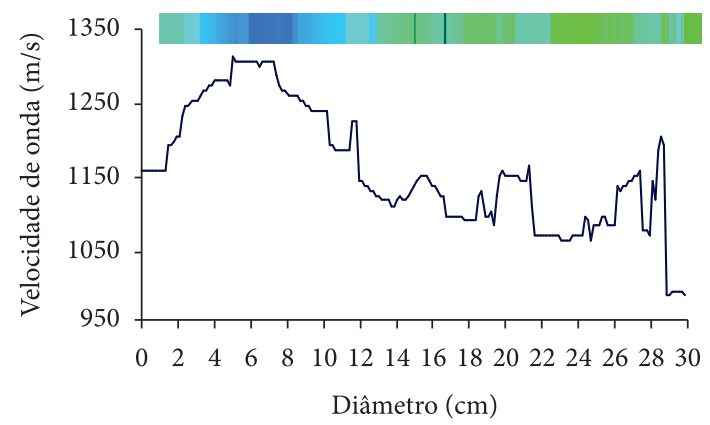



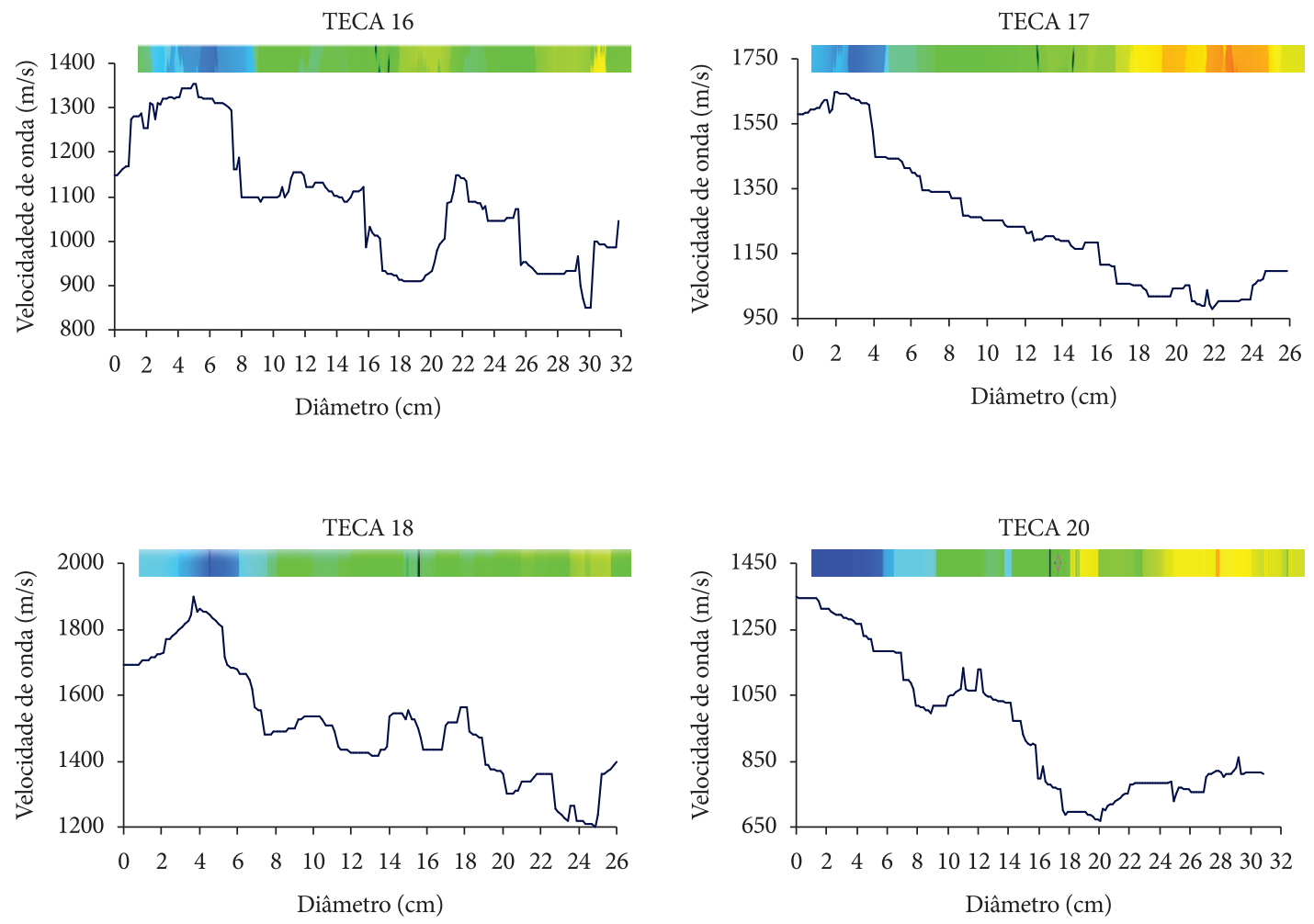

TECA 21
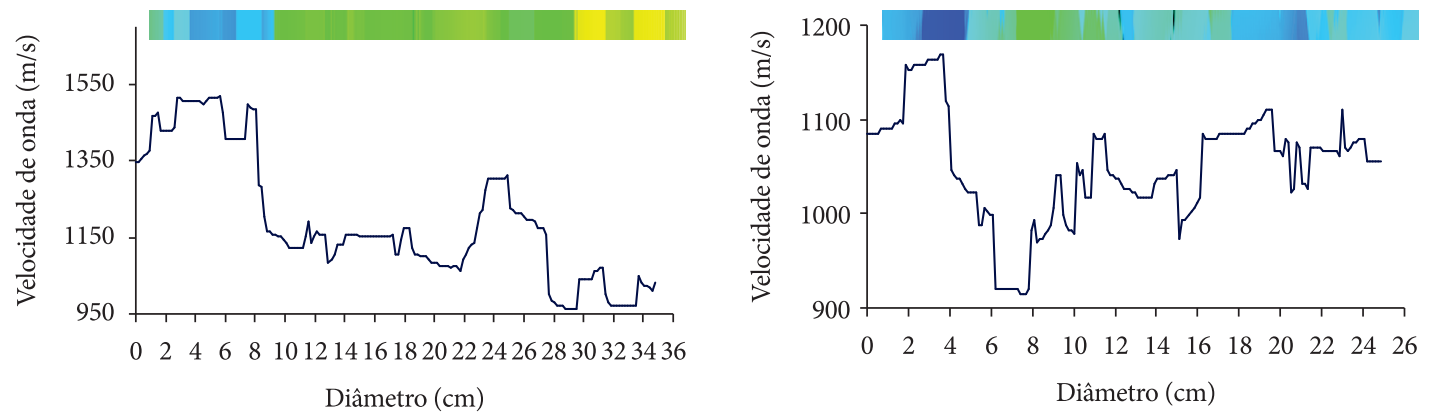

TECA 23
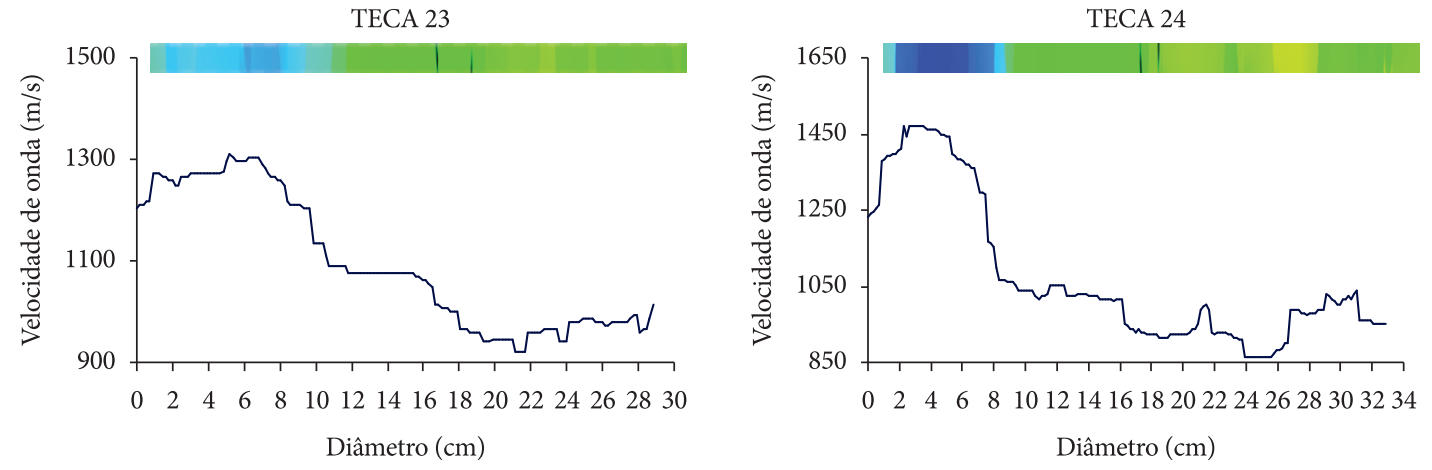

Figura 3. Continuação...

Figure 3. Continued... 
TECA 29

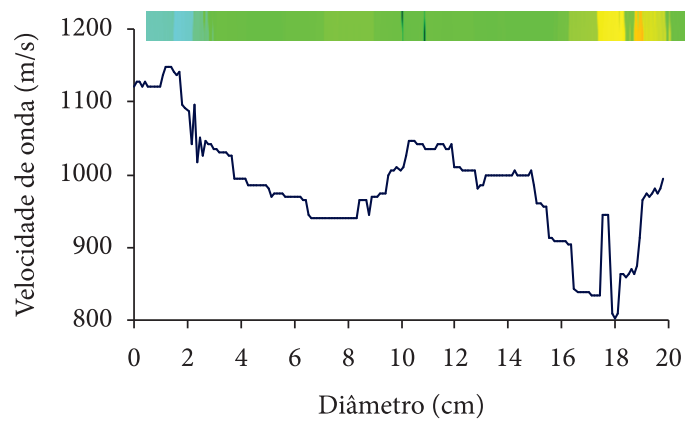

TECA 31

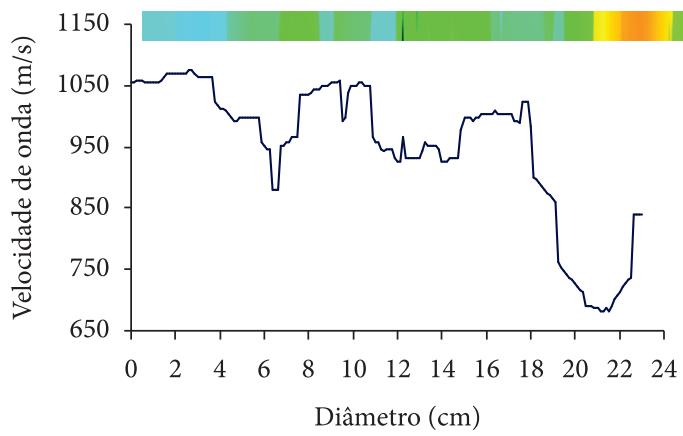

TECA 30

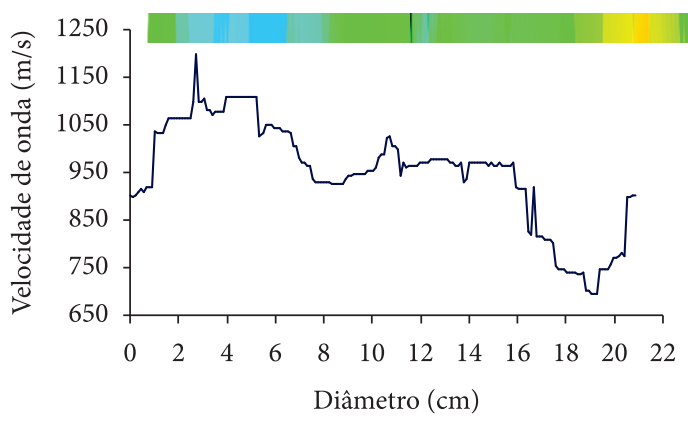

TECA 32

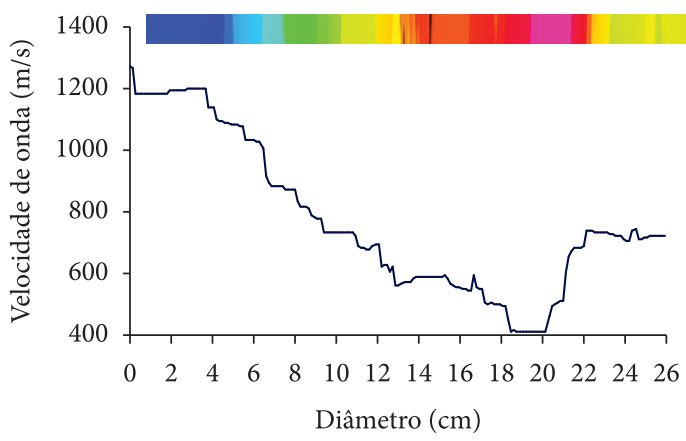

Figura 3. Continuação...

Figure 3. Continued...

Tabela 1. Valores de velocidade de onda no lenho das árvores de teca.

Table 1. Values of wave velocity in the wood of teak trees.

\begin{tabular}{|ccrrrrr|} 
Árvore & $\begin{array}{c}\text { Diâmetro } \\
(\mathbf{c m})\end{array}$ & Média & $\begin{array}{c}\mathbf{C V}^{*} \\
\mathbf{( \% )}\end{array}$ & $\begin{array}{c}\text { Desvio } \\
\text { padrão }\end{array}$ & Mínimo & Máximo \\
\hline 3 & 33 & 1038,61 & 7,43 & 77,21 & 911 & 1239 \\
\hline 5 & 27 & 1064,20 & 14,84 & 157,96 & 878 & 1368 \\
\hline 6 & 23 & 1114,97 & 10,73 & 119,60 & 817 & 1286 \\
\hline 7 & 20 & 1145,40 & 9,10 & 104,27 & 796 & 1258 \\
\hline 8 & 20 & 1070,05 & 3,12 & 33,42 & 1007 & 1131 \\
\hline 10 & 25 & 1392,23 & 19,33 & 269,10 & 1003 & 1906 \\
\hline 12 & 33 & 1057,59 & 11,86 & 125,38 & 882 & 1289 \\
\hline 13 & 30 & 1161,85 & 6,98 & 81,10 & 991 & 1314 \\
\hline 16 & 32 & 1090,71 & 12,66 & 138,10 & 849 & 1352 \\
\hline 17 & 26 & 1241,64 & 16,50 & 204,82 & 980 & 1650 \\
\hline $\mathbf{1 8}$ & $\mathbf{2 6}$ & $\mathbf{1 5 0 9 , 6 9}$ & $\mathbf{1 1 , 4 3}$ & $\mathbf{1 7 2 , 5 8}$ & $\mathbf{1 2 0 0}$ & $\mathbf{1 8 9 9}$ \\
\hline 20 & 31 & 954,79 & 22,01 & 210,18 & 669 & 1350 \\
\hline 21 & 36 & 1195,54 & 13,88 & 165,89 & 964 & 1521 \\
\hline 22 & 25 & 1049,00 & 5,51 & 57,75 & 915 & 1168 \\
\hline 23 & 29 & 1094,44 & 11,86 & 129,85 & 919 & 1311 \\
\hline 24 & 33 & 1073,38 & 17,41 & 186,83 & 866 & 1470 \\
\hline 29 & 20 & 985,40 & 7,58 & 74,73 & 803 & 1147 \\
\hline 30 & 21 & 949,38 & 11,62 & 110,34 & 696 & 1200 \\
\hline 31 & 24 & 950,86 & 11,96 & 113,72 & 681 & 1075 \\
\hline 32 & $\mathbf{2 6}$ & $\mathbf{7 7 2 , 1 2}$ & $\mathbf{3 1 , 9 9}$ & $\mathbf{2 4 7 , 0 0}$ & $\mathbf{4 1 3}$ & $\mathbf{1 2 7 1}$ \\
\hline Total & & 1087,13 & 19,41 & 211,04 & 413 & 1906 \\
\hline
\end{tabular}

*Coeficiente de variação. 
Tabela 2. Frequências para as velocidades de onda $(\mathrm{m} / \mathrm{s})$ no lenho das árvores de teca.

Table 2. Frequencies for wave velocities $(\mathrm{m} / \mathrm{s})$ in the wood of teak trees.

\begin{tabular}{|ccccc|}
$\begin{array}{c}\text { Velocidade de onda } \\
(\mathbf{m} / \mathbf{s})\end{array}$ & $\begin{array}{c}\text { Frequência } \\
\text { absoluta }\end{array}$ & $\begin{array}{c}\text { Frequência } \\
\text { relativa } \%\end{array}$ & $\begin{array}{c}\text { Frequência } \\
\text { acumulada }\end{array}$ & $\begin{array}{c}\text { Frequência } \\
\text { Rel. Acum. }\end{array}$ \\
\hline $407-507$ & 26 & 0,73 & 26 & 0,0073 \\
\hline $507-607$ & 35 & 0,98 & 61 & 0,0171 \\
\hline $607-707$ & 55 & 1,54 & 116 & 0,0325 \\
\hline $707-807$ & 132 & 3,70 & 248 & 0,0695 \\
\hline $807-907$ & 167 & 4,68 & 415 & 0,1163 \\
\hline $907-1007$ & 832 & 23,32 & 1247 & 0,3496 \\
\hline $1007-1107$ & 940 & 26,35 & 2187 & 0,6131 \\
\hline $1107-1207$ & 598 & 16,76 & 2785 & 0,7808 \\
\hline $1207-1307$ & 372 & 10,43 & 3157 & 0,8851 \\
\hline $1307-1407$ & 156 & 4,37 & 3313 & 0,9288 \\
\hline $1407-1507$ & 99 & 2,78 & 3412 & 0,9565 \\
\hline $1507-1607$ & 66 & 1,85 & 3478 & 0,9750 \\
\hline $1607-1707$ & 43 & 1,21 & 3521 & 0,9871 \\
\hline $1707-1807$ & 23 & 0,64 & 3544 & 0,9936 \\
\hline $1807-1907$ & 23 & 0,64 & 3567 & 1,0000 \\
\hline
\end{tabular}

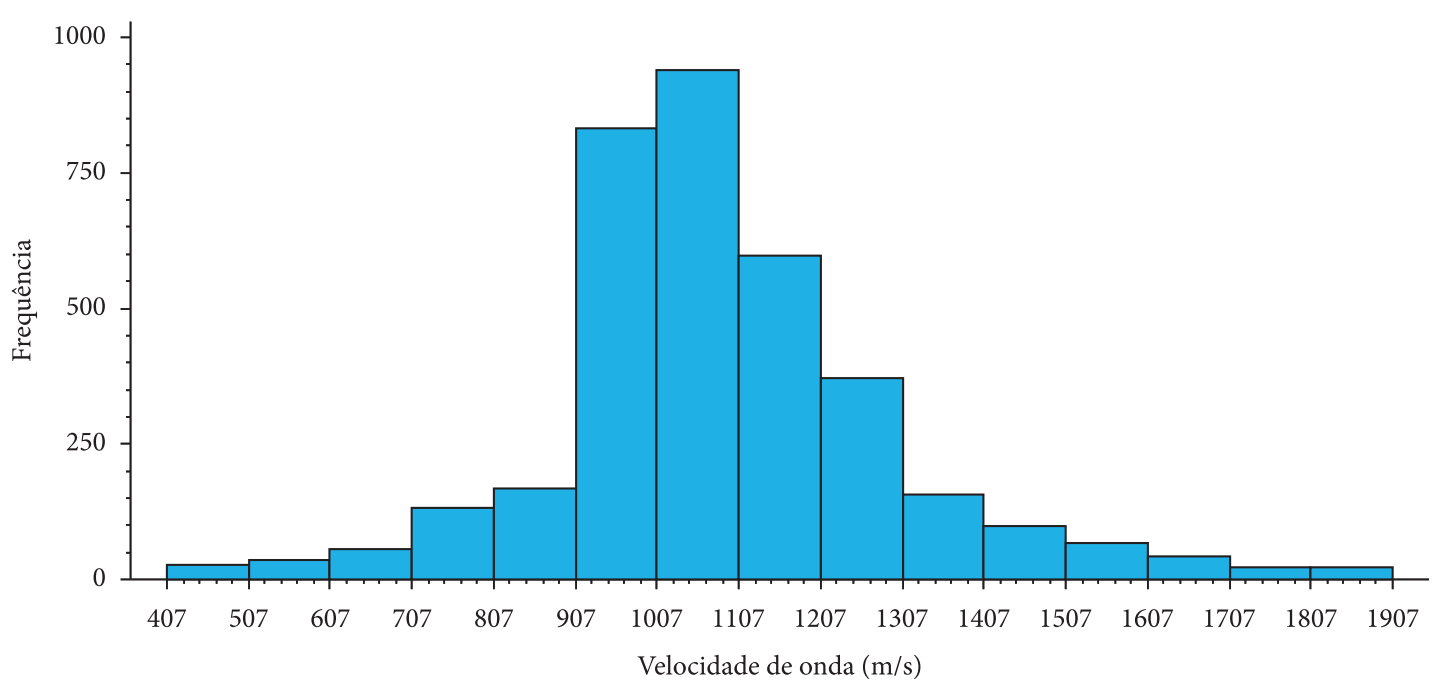

Figura 4. Histograma de frequência da velocidade de onda no lenho das árvores de teca.

Figure 4. Histogram of frequency of the wave velocity in the trunk of teak trees.

Apenas 3,25, 0,73 e 0,64\% das árvores apresentam valores de velocidade de onda no lenho de 407-707, 407-507 e de 1807-1907 m/s, respectivamente. Por outro lado, em 76,86\% das árvores, observa-se a maior frequência de leituras de velocidade de 907-1307 m/s, dado verificado claramente no histograma de frequência da velocidade de onda através do lenho das árvores (Figura 4).

\section{CONCLUSÕES}

Os resultados do presente trabalho permitem concluir que a aplicação da técnica de tomografia de impulso permitiu: i) analisar o estado de sanidade e das propriedades do lenho, pelo exame das imagens da seção transversal do tronco; e ii) que o perfil radial foi eficaz na avaliação da integridade do lenho 
das árvores de teca. O equipamento e a metodologia mostraram-se potenciais na avaliação não destrutiva do lenho de árvores em florestas urbanas e em plantações florestais para aplicação em programas de manejo, seleção genética e qualidade do lenho.

\section{STATUS DA SUBMISSÃO}

Recebido: 15/12/2010

Aceito: 15/04/2011

Resumo publicado online: 20/04/2011

Artigo completo publicado: 30/06/2011

\section{AUTOR(ES) PARA CORRESPONDÊNCIA}

\section{Vinicius Resende de Castro}

Departamento de Ciências Florestais, Escola Superior de Agricultura "Luiz de Queiroz" ESALQ, Universidade de São Paulo - USP, Av. Pádua Dias, 11, CP 9, CEP 13418-900, Piracicaba, SP, Brasil e-mail:vresende@gmail.com

\section{REFERENNCIAS}

Amodei JB, Oliveira BRU, Gurgel M M, Medeiros RA, Latorraca JVF. Avaliação tomográfica de árvores de Tectona grandis L. f. para avaliação preliminar da qualidade da madeira. In: Anais do II Simpósio de Ciência e Tecnologia da Madeira do Rio de Janeiro; 2010;
Rio de Janeiro. Rio de Janeiro: Universidade Federal Rural do Rio de Janeiro; 2010. CD-ROM.

Ferreira ATB. Caracterização da estrutura anatômica do lenho, dos anéis de crescimento e dos canais de resina de árvores de Pinus caribaea var. hondurensis Barr. et Golf. [dissertação]. Piracicaba: Escola Superior de Agricultura "Luiz de Queiroz", Universidade de São Paulo; 2009.

Gilbert EA, Smiley T. Picus Sonic tomography for the quantification of decay in white oak (Quercus alba) and hickory (Carya spp.). Journal of Arboriculture 2004; 30(5):277-281.

Pereira LC, Silva Filho DF, Tomozelo Filho M, Couto HTZ, Moreira JMMÁP, Polizel JL. Tomografia de impulso para avaliação do interior do lenho de árvores. Revista da Sociedade Brasileira de Arborização Urbana 2007; 2(2):65-75

Pereira LCR. Tomografia de impulso para estimativa da densidade da madeira [dissertação]. Piracicaba: Escola superior de Agricultura "Luiz de Queiroz", Universidade de São Paulo; 2009.

Picus. Picus home page. [cited 2009 set. 30]. Available from: http://www.argus-electronic.de/index.php/en/ picus-sonic-tomograph/faq-sonic.

Rinntech. User Manual - Arbotom 3-D Tree Impulse Tomograph, version 1.59 for Microsoft Windows 98, 2000, XP. Heidelberg: Microsoft Window; 2005. 42 p.

Rollo FMA. Identificação de padrões de resposta à tomografia de impulso em tipuanas (Tipuana tipu (Benth.) O. Kuntze [dissertação]. Piracicaba: Escola superior de Agricultura "Luiz de Queiroz", Universidade de São Paulo; 2009. 